\title{
Determination of parameters of cascade showers in the water calorimeter using 3D-distribution of Cherenkov light
}

\author{
R.P. Kokoulin ${ }^{1}$, A.G. Bogdanov, S.S. Khokhlov, V.A. Khomyakov, V.V. Kindin, \\ A.A. Petrukhin, V.V. Shutenko, I.I. Yashin \\ National Research Nuclear University MEPhI (Moscow Engineering Physics Institute) \\ Moscow 115409, Russia \\ E-mail: RPKokoulin@mephi.ru
}

\begin{abstract}
Results of investigations of cascade showers produced by muons in Cherenkov water calorimeter (CWC) NEVOD with a dense lattice of quasi-spherical measuring modules are presented. The spatial distribution of the Cherenkov radiation from the cascades is obtained. It represents the dependence of the radiation intensity on the depth of the shower development and on the distance from its axis with a step of 0.5 meter in both directions. The obtained results allowed to develop methods for selecting the cascades among the events with a high energy release and for estimating the parameters of cascades on the basis of the CWC data. The accuracy of such estimations is considered. The zenith angular distribution of experimental events with cascades is presented, and the problem of separation of cascades produced by hadrons and by muons is discussed. The energy spectrum of showers with energies from 100 $\mathrm{GeV}$ to $15 \mathrm{TeV}$ generated by muons is measured.
\end{abstract}




\section{Introduction}

Cherenkov water detectors are one of the widely used tools for studies of ultrahigh-energy muons and neutrinos. Energy spectra of these particles are determined on the basis of the spectra of cascade showers generated by them in water. To determine the characteristics of showers by means of the response of the detector, different models are used. But not all of them are experimentally verified. Thus the study of distribution of Cherenkov light from cascade showers in water is a topical experimental problem. Its solution allows to improve approaches to the problems of selecting cascades and reconstructing their parameters; it also puts these approaches on a solid experimental basis.

Till present, a detailed experimental study of the image of the shower in Cherenkov light was not carried out. In existing large Cherenkov facilities such as ANTARES [1], IceCube [2], Baikal [3] the distances between optical modules multiply exceed the dimensions of the detected showers. So the showers are usually considered in a point approximation; obtainment of a detailed picture of the shower in Cherenkov light is almost impossible. In the present work, the spatial distribution of Cherenkov radiation from the cascade showers was studied on the basis of the experimental complex NEVOD [4] (figure 1), which includes the Cherenkov water calorimeter (CWC) with a dense spatial lattice of measuring modules. It allows to investigate showers in details, which are inaccessible for large Cherenkov detectors.

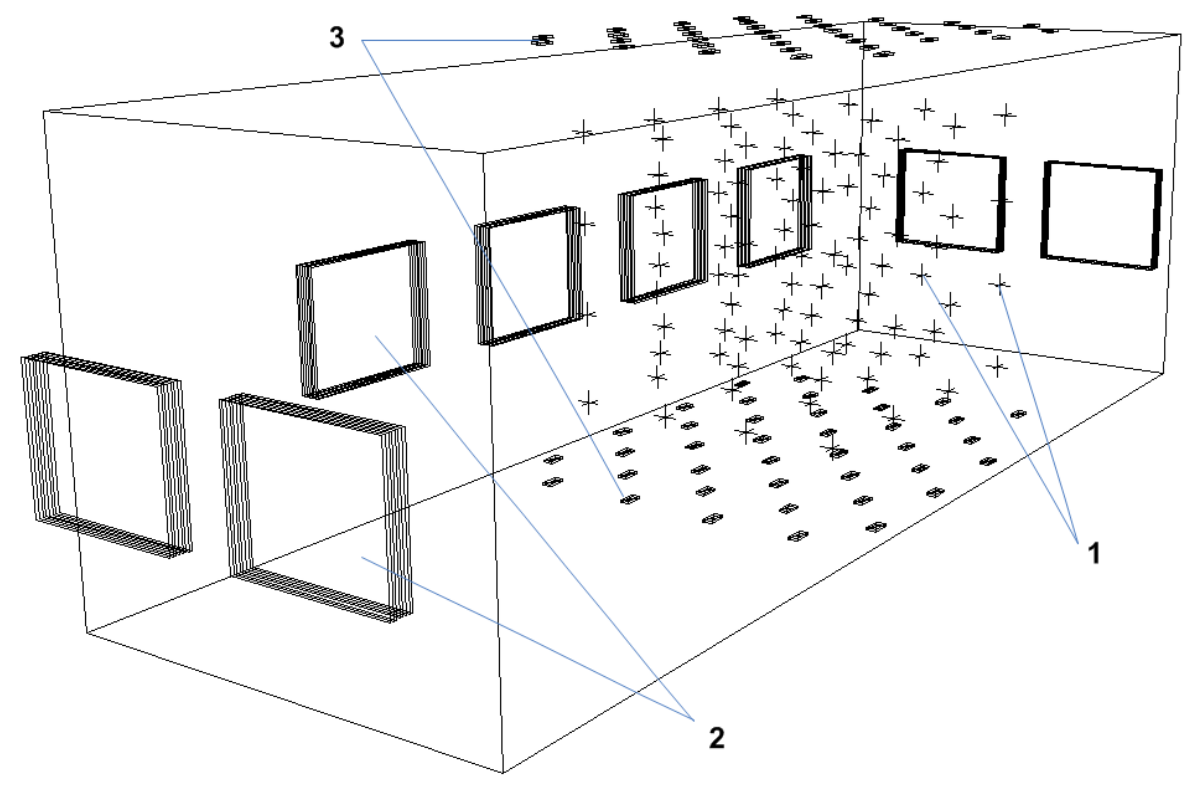

Figure 1: Layout of basic facilities of the experimental complex NEVOD: 1 - quasi-spherical modules of CWC, 2 - supermodules of the coordinate-tracking detector DECOR, 3 - detectors of the system of calibration telescopes.

CWC NEWOD with size $9 \times 9 \times 26 \mathrm{~m}^{3}$ is equipped with the lattice of 91 quasi-spherical modules (QSM), which are located with steps of $2.5 \mathrm{~m}$ along the detector, $2 \mathrm{~m}$ across it and $2 \mathrm{~m}$ in depth. Each QSM includes six FEU-200 photomultipliers with flat $15 \mathrm{~cm}$ diameter photocathodes oriented along the axes of the rectangular coordinate system. Such design of the module allows to measure the intensity of Cherenkov radiation from relativistic charged particles with almost equal sensitivity for all directions of incident light and allows to reconstruct this direction. 
High density of the lattice and a wide dynamic range of detected signals $\left(1-10^{5}\right.$ photoelectrons for each PMT [5]) provide a capability for measuring the spatial distribution of Cherenkov light from showers with a small step (minimal step is $0.5 \mathrm{~m}$ that corresponds to the size of the module). Such measurements were carried out for events with showers in which a near-horizontal muon generated a shower in the sensitive volume of the detector. The tracks of the muons in such events were determined according to the data from the supermodules of the coordinate-tracking detector DECOR [6], which are located in the galleries along the short sides of the CWC. The zenith angles of the muons in these events are in the range of $85^{\circ}-90^{\circ}$, average energy of such muons is about $100 \mathrm{GeV}$. It was assumed that the track of the muon coincides with the axis of the shower, because the RMS deviation of the trajectory from a straight line at the distance of 26 meters in water for such muons is rather small (less than $1 \mathrm{~cm}$ ).

Reconstruction of parameters of the showers was made according to the earlier proposed method [7] and with an assumption that all cascade electrons move along the axis of the shower and emit photons at an angle of about $42^{\circ}$. To measure the cascade curve, the axis of the shower within the CWC was divided into bins one radiation length each $\left(36.1 \mathrm{~g} \mathrm{~cm}^{-2}\right.$ in water). The responses from the PMTs which «see» the radiation from a bin at the angle of Cherenkov radiation were selected. The response of each PMT was converted to the number of charged cascade particles in the corresponding bin. In the case of several PMTs for one bin, the results were averaged taking into account the errors. The energy of the shower and its point of maximal development were determined by fitting the experimental cascade curve with K. Greisen's onedimensional analytical approximation of the number of particles vs depth [8].

\section{3D-distribution of Cherenkov radiation from cascades}

Spatial distribution of the Cherenkov radiation was measured for showers with reconstructed energies from 100 to $500 \mathrm{GeV} ; 522$ cascades were selected from the experimental data series for the period 2013-2015 with about 12 thousand hours of «live» time. This set of cascades was hereinafter used as a reference set.

The dependence of the response of QSM on the distance from the axis and on the depth of shower development was measured. The point of maximum shower development $t_{\max }$ was taken as the zero point on the axis of depth. The root of the sum of squares of responses of PMTs was used as the response of QSM (this value weakly depends on the direction of incident light [9]). The responses of QSMs were also normalized on the energy of the shower:

$$
B=\frac{\varepsilon_{0}}{\varepsilon} \sqrt{\sum A_{i}^{2}},
$$

where $\varepsilon_{0}$ is equal to $200 \mathrm{GeV}$ (close to the average energy of the showers in the reference set); $\varepsilon$ is the reconstructed energy of the shower in the event; $A_{\mathrm{i}}$ is the response of the $\mathrm{i}$-th PMT in the QSM (in photoelectrons, p.e.).

The dependence of $B$ on the depth and distance from the axis of the shower was obtained by averaging for all events of the reference set. It is presented in figure $2 \mathrm{a}$. The obtained dependence is mainly determined by two factors. The first of them is the attenuation of the Cherenkov light in water as a result of absorption and cylindrical divergence. The second one is the directional divergence of the light caused by multiple scattering of cascade particles. 
The influence of the energy distribution of cascade particles is less significant factor and can be ignored.

In order to study the angular distribution of cascade particles, the ratio of the intensity of the light measured for cascades $(B)$ to the intensity for single muons $\left(B_{\mu}\right)$ was considered. It was made according to the assumption that the parameters of light attenuation are the same for showers and for single muons. The distribution of this ratio is presented in figure $2 b$.
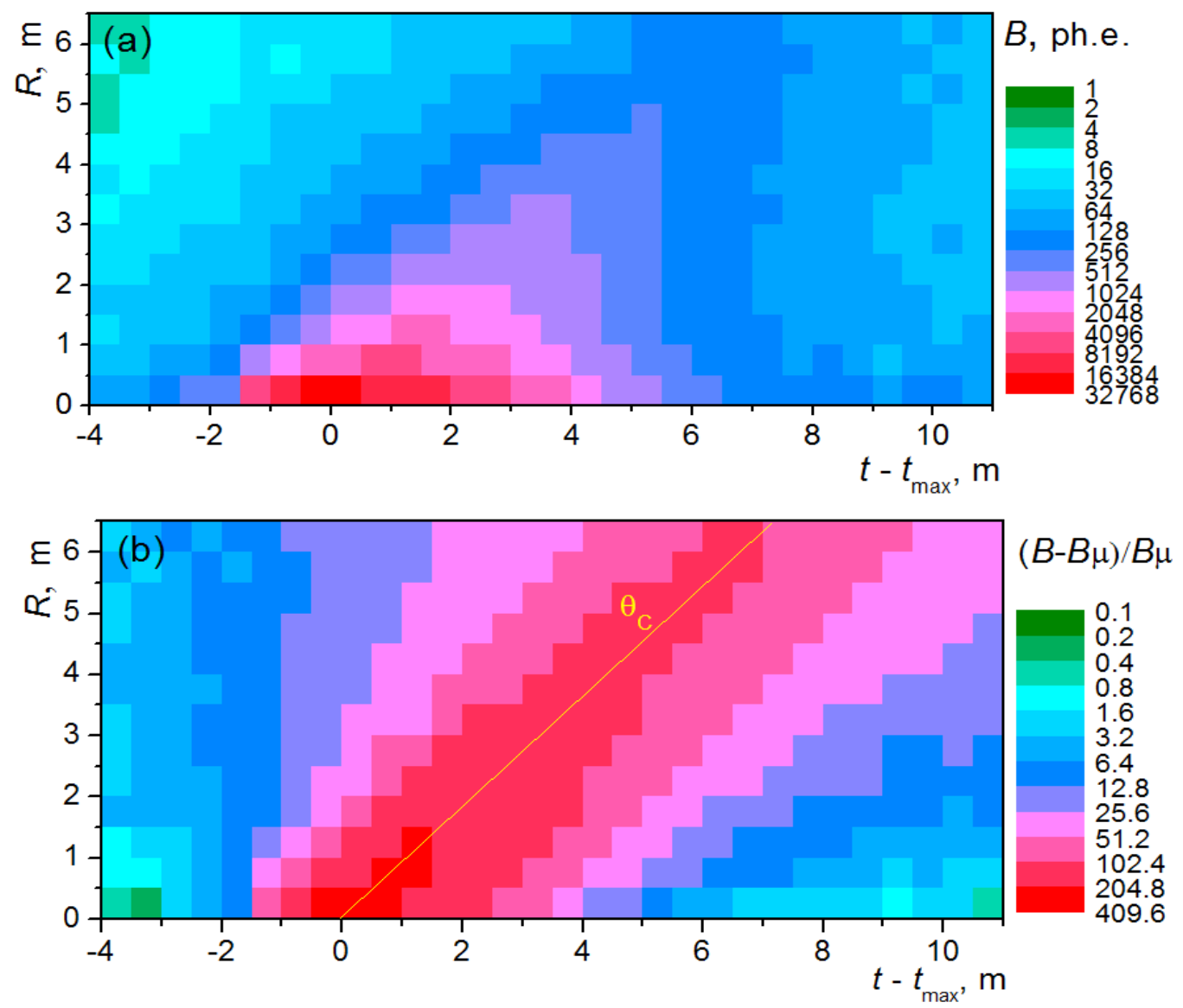

Figure 2: Response of CWC to a cascade depending on the depth $t-t_{\max }$ of the shower development and on the distance $R$ from the axis of the shower (a); ratio between the responses to a cascade and to a single muon (b). A straight line represents the Cherenkov angle for water.

The figure $2 b$ shows the shift of the maximum intensity of Cherenkov radiation with depth that corresponds, as confirmed by the analysis, to the Cherenkov angle. The observed broadening of the areas of equal intensity with increasing distance from the axis of the shower is associated with the angular divergence of cascade particles.

Calculations of spatial distribution of the Cherenkov radiation were conducted for two models of angular distribution of cascade particles. One of them is the analytical calculation of the I.P. Ivanenko group, based on the cascade equations [10]. Another one is the approximation obtained by the participants of the IceCube collaboration [11] of results of simulation with GEANT 3 performed by C. Wiebusch [12]. The comparison of the experimental and calculated distributions of the Cherenkov light showed that both models are in satisfactory agreement with the experimental results [13]. However, for the backward hemisphere (relative to the direction of the shower) a better agreement gives the model of C. Wiebusch. 


\section{Methods of selecting cascade showers and reconstructing their parameters}

The obtained results allowed to develop the algorithm of iterative reconstruction of the parameters of cascade showers. It uses the responses of quasi-spherical modules of CWC only. The number of parameters in the reconstruction procedure increases to six, because the direction of the axis of the shower and its location are unknown. The calculation of the expected values of the responses of QSMs was based on the following models and assumptions. The Greisen approximation was used to determine the number of cascade particles depending on the depth of the shower development. The angular distribution of the Cherenkov radiation was determined on the basis of the Wiebusch model of the angular distribution of cascade electrons. The experimentally measured response of modules of CWC NEVOD in events with single muons (adjusted for the ratio of the average energy losses for muons and electrons in water) was used as a response to a single particle [14].

The estimates based on the data of a group of QSMs with the highest responses were used to determine the initial values of the unknown parameters. The calibration of methods was conducted on the data of the reference set of events. Simple calorimetric estimate based on the total response of such QSMs was used for evaluation of the energy. The location of maximum of shower development was estimated as the center of gravity of the group of QSMs taking into account their responses. The direction of the axis of shower was assessed according to the responses of PMTs of the modules in the selected group: responses of PMTs taking into account their spatial orientation indicate the direction of the axis of the shower. Afterward, the best fit parameters were found iteratively by means of the least squares method.

Verification of proposed algorithm was carried out using the showers with known axes that were determined according to the DECOR data (the reference set). Figure 3 shows the obtained results for reconstruction of the energy of the shower and its direction:

$$
y_{0}=\ln \left(\frac{\varepsilon_{\mathrm{rec}}}{\beta}\right), \beta=78 \mathrm{MeV}
$$
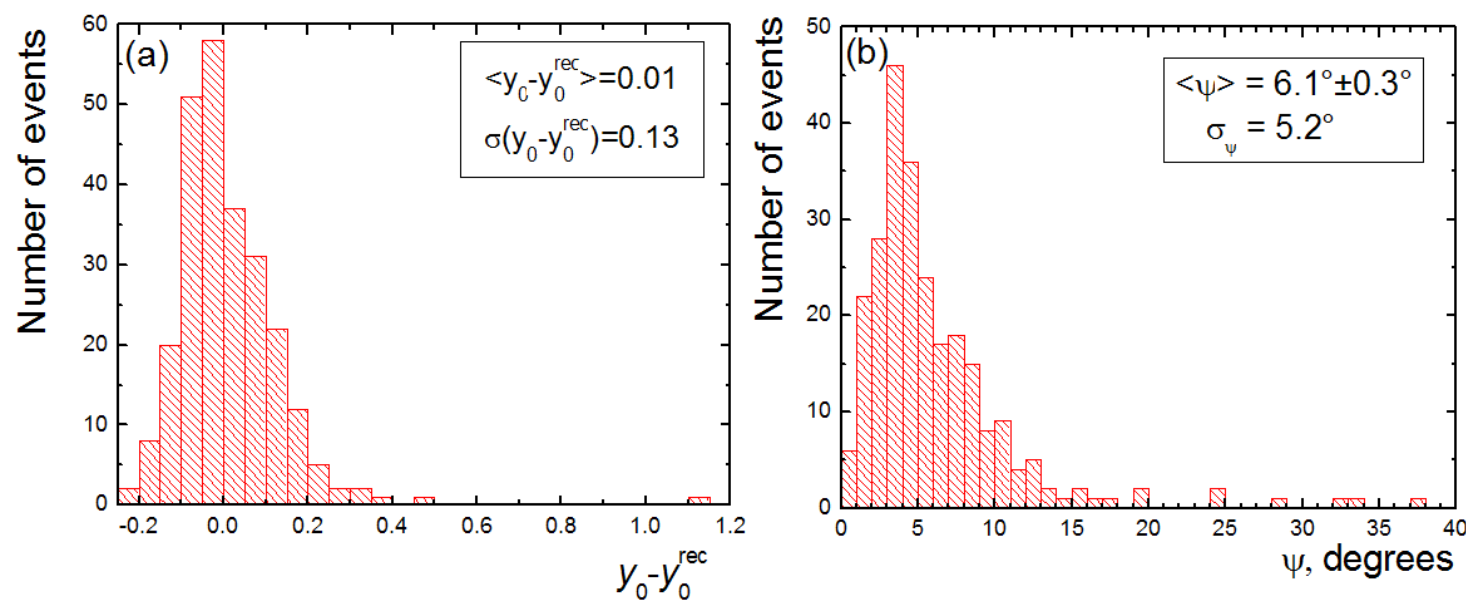

Figure 3: Comparison of results of reconstruction of the energy parameter $y_{0}$ of the shower for methods with known and unknown axis (a); distribution for error of reconstructed direction of the axis of the shower (b). 
The errors of reconstructed parameters of the shower according to the presented distributions are about $6^{\circ}$ for direction of the axis and about $13 \%$ for the energy in the interval $\varepsilon>100 \mathrm{GeV}$.

Criterion for selection of cascade showers among the events with a high energy deposit was developed. It is based on the assumption that in the case of the birth of the cascade shower in the sensitive volume of the Cherenkov detector, the group of QSMs with highest responses forms a compact spatial cluster. The criterion uses the value of mean square radius of the cluster of modules with highest responses. The threshold value is chosen according to the reference set; it equals to $2.4 \mathrm{~m}$ for 10 brightest QSMs.

Criteria that provide stable reconstruction of parameters were also developed. The analysis of the simulated data showed that characteristics of the showers are sustainably reconstructed only in the events where the shower develops in the sensitive volume of the detector. When the cascade shower is located outside of this volume, the peripheral QSMs of the lattice of detecting modules have the highest responses. Such events are discarded.

About 360 thousand showers with reconstructed energy more than $100 \mathrm{GeV}$ were selected in the data of experimental series with duration of 11.9 thousand hours of «live» time. Figure 4 shows the zenith angular distribution of these events. It also presents the calculation of the zenith angular distribution of showers with such energies that were generated exclusively by muons. Previously obtained distribution for the error of angular reconstruction of the shower axis was taken into account in calculations for a proper comparison with the experimental data.

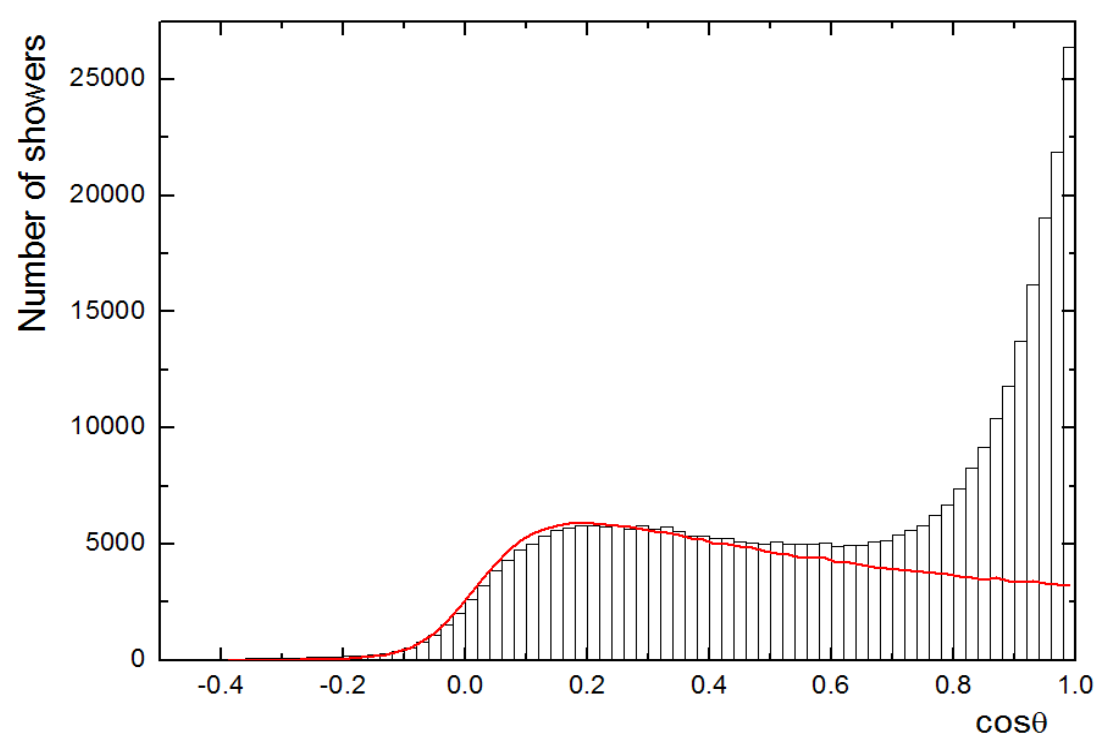

Figure 4: Zenith angular distribution of cascade showers: histogram represents experimental data; curve is calculation for cascade showers generated by muons corrected for the error of the reconstruction of the axis direction.

There is a good agreement between experimental and calculated data. The comparison of diagrams for all the showers and for the showers generated exclusively by muons shows that for zenith angles greater than $55^{\circ}$ (the value of the cosine of the angle of less than 0.57 ) the fraction of showers generated by hadrons does not exceed $2-3 \%$. In accordance to this conclusion, a criterion for the selection of showers from muons based on the value of reconstructed zenith angle of the axis $\left(\theta>55^{\circ}\right)$ was used. 
The number of experimental events that passed this criterion (i.e. showers generated by muons) is about 150 thousand. According to these data, a preliminary differential energy spectrum of showers with energies from $100 \mathrm{GeV}$ to $15 \mathrm{TeV}$ was constructed. The result is shown in figure 5. The spectrum of the showers with axes determined according to the coordinate detector DECOR and the calculations of the expected spectrum for different slopes $\gamma$ of generation function of parent pions and kaons are also presented for the comparison.

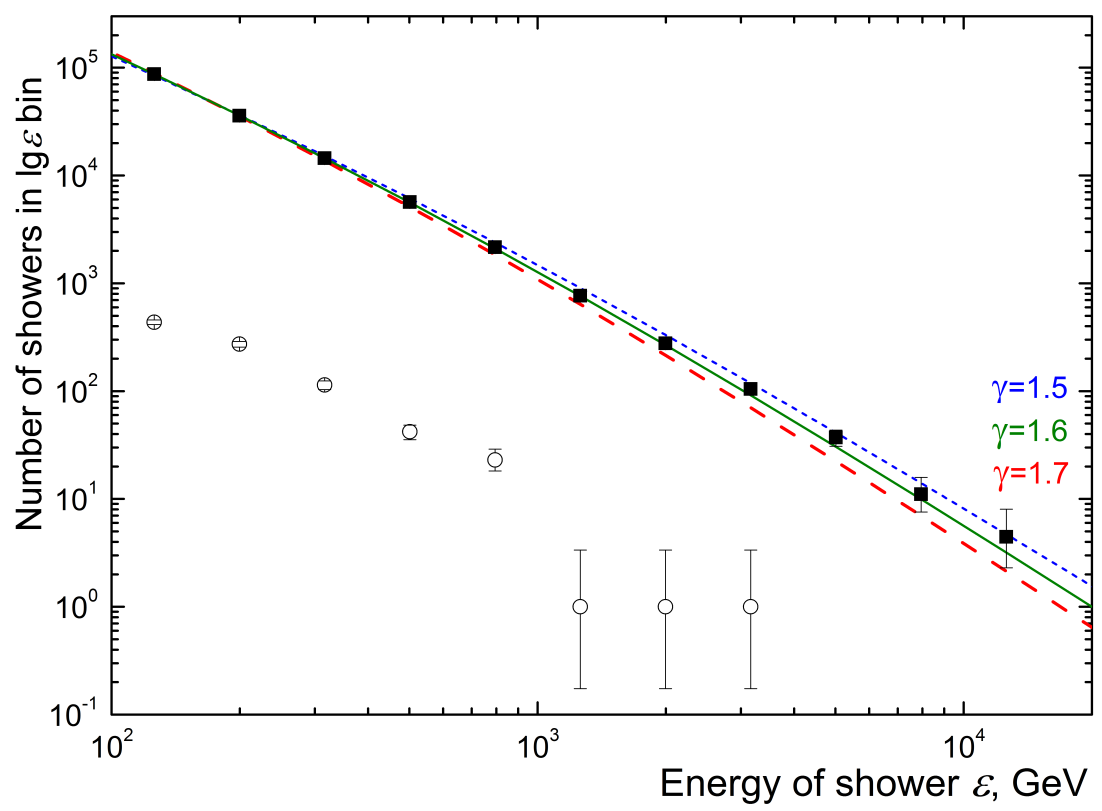

Figure 5: Differential energy spectrum of cascade showers generated by muons in water. Squares: present experiment (zenith angles $55^{\circ}-90^{\circ}$ ); circles: experiment [14] for cascades with axes reconstructed from DECOR data; curves: calculations for $\gamma=1.5,1.6$ and 1.7.

The analysis showed that for the assumption of a constant slope $\gamma$ of generation function of parent pions and kaons in the considered energy range the experimental data correspond to the value of $\gamma$ close to 1.6 .

\section{Conclusions}

The spatial distribution of Cherenkov radiation from cascade showers with energies $100-$ $500 \mathrm{GeV}$ generated by muons is measured for the first time in the Cherenkov water calorimeter NEVOD with a dense lattice of quasi-spherical measuring modules. The obtained results provided the development of criteria for selecting the cascade showers in the events with a high energy deposit and methods of reconstruction of their parameters. The average value of error of reconstructed energy of the shower is about $13 \%$, the error in determining the direction of the axis of shower is approximately $6^{\circ}$. The events with cascades were selected in the data of a long-term experimental series, and the parameters of these cascades were reconstructed. The analysis of the zenith angular distribution of these events allowed to set the criterion for separation of cascades from muons and hadrons. Finally, the differential energy spectrum of showers with energies from $100 \mathrm{GeV}$ to $15 \mathrm{TeV}$ generated by muons in the range of zenith angles more than $55^{\circ}$ was measured. 
This work was performed at the Unique Scientific Facility Experimental complex NEVOD. It was supported by the RF Ministry of Education and Science (contract RFMEFI59114X0002, MEPhI Academic Excellence Project, and government task) and by the Russian Foundation for Basic Research, grant No. 15-02-07763.

\section{References}

[1] S. Mangano (ANTARES Collaboration), Nucl. Instr. and Meth. in Phys. Res. A, 588, 107 (2008).

[2] M.G. Aartsen et al. (IceCube Collaboration), J. Instr., 9, 03009 (2014).

[3] G.V. Domogatsky, Physics Uspekhi, 54, 949 (2011).

[4] V.M. Aynutdinov et al., Astrophysics and Space Science, 258, 105 (1997).

[5] S.S. Khokhlov et al., Bull. Leb. Phys. Inst., 37, 126 (2010).

[6] N.S. Barbashina et al., Instrum. Exp. Tech., 43, 743 (2000).

[7] S.S. Khokhlov et al., Bull. Russ. Acad. Sci. Phys., 77, 638 (2013).

[8] S. Hayakawa, Cosmic Ray Physics, NY: J. Wiley \& Sons (1969).

[9] V.A. Khomyakov et al., Physics Procedia, 74, 442 (2015).

[10] A.A. Belayev, I.P. Ivanenko, V.V. Makarov, Proc. 1978 DUMAND Summer Workshop, vol. 1, 337 (1978).

[11] M.G. Aartsen et al., Nucl. Instr. and Meth. in Phys. Res. A, 711, 73 (2013).

[12] C.H. Wiebusch, Ph.D. Thesis, Physikalische Institute RWTH Aachen (1995).

[13] V.A. Khomyakov et al., Bull. Russ. Acad. Sci. Phys., 81, 490 (2017).

[14] R.P. Kokoulin et al., Proceedings of Science. PoS(ICRC2015)360. 